\title{
Letter to the Editor: "Incidence of contralateral occult inguinal hernia found at the time of laparoscopic trans-abdominal pre-peritoneal (TAPP) repair" by Griffin et al. (Hernia 14:345-349, 2010)
}

\author{
K. J. P. van Wessem • P. W. Plaisier • \\ J. F. Lange
}

Received: 15 September 2010/Accepted: 3 October 2010/Published online: 13 October 2010

(C) The Author(s) 2010. This article is published with open access at Springerlink.com

\section{Dear Editor,}

We read with great interest the recent article by Griffin et al. [1]. The authors studied the incidence of occult contralateral hernias during trans-abdominal pre-peritoneal (TAPP) repair and found an incidence of $22 \%$ in patients scheduled for unilateral repair. We would like to congratulate the authors on their work, but we have some criticisms to be discussed.

We noticed that a distinct definition of 'occult hernia' is lacking. Did the authors actually mean a patent processus vaginalis (PPV) or did they find a true herniation of abdominal content through the open processus? If it really concerned herniations, we missed the differentiation between direct and indirect hernias. Moreover, it must be concluded, then, that $22 \%$ of the patients had been misdiagnosed by clinical evaluation. We, therefore, expect the authors to mean PPV when using the term 'occult hernia.'

We ourselves have studied PPV extensively. In an adult population undergoing laparoscopic surgery for treatment other than hernia repair, we found an incidence of $12 \%$ [2]. In a follow up study on the same study population 5 years later, we also found that patients with a PPV have a higher

K. J. P. van Wessem ( $\square)$

Department of Surgery, University Medical Center Utrecht, Heidelberglaan 100, 3584 CX Utrecht, The Netherlands e-mail: K.J.P.Wessem@umcutrecht.nl

P. W. Plaisier

Department of Surgery, Albert Schweitzer Hospital, Dordrecht, The Netherlands

J. F. Lange

Department of Surgery, Erasmus Medical Center,

Rotterdam, The Netherlands chance of developing an indirect hernia than patients with an obliterated processus vaginalis, 12 vs. $3 \%$ [3].

Since the majority of people with PPV will not develop hernias, whereas some people without PPV will, we think that the relationship between a PPV and the development of a hernia is not at all crystal clear. We, therefore, feel that care should be taken when casually repairing asymptomatic PPVs just because the contralateral side is being repaired. In this respect, it also has to be considered that a significant proportion of hernias, if having developed at all, will remain asymptomatic and will probably need no operation at all. Finally, the operating times are longer, the costs are higher, and, as with every operation, laparoscopic hernia repair is still not without complications [4].

Open Access This article is distributed under the terms of the Creative Commons Attribution Noncommercial License which permits any noncommercial use, distribution, and reproduction in any medium, provided the original author(s) and source are credited.

\section{References}

1. Griffin KJ, Harris S, Tang TY, Skelton N, Reed JB, Harris AM (2010) Incidence of contralateral occult inguinal hernia found at the time of laparoscopic trans-abdominal pre-peritoneal (TAPP) repair. Hernia 14:345-349

2. van Wessem KJ, Simons MP, Plaisier PW, Lange JF (2003) The etiology of indirect inguinal hernias: congenital and/or acquired? Hernia 7:76-79

3. van Veen RN, van Wessem KJ, Halm JA, Simons MP, Plaisier PW, Jeekel J, Lange JF (2007) Patent processus vaginalis in the adult as a risk factor for the occurrence of indirect inguinal hernia. Surg Endosc 21:202-205

4. Lovisetto F, Zonta S, Rota E, Bottero L, Faillace G, Turra G, Fantini A, Longoni M (2007) Laparoscopic transabdominal preperitoneal (TAPP) hernia repair: surgical phases and complications. Surg Endosc 21:646-652 